\title{
On the distribution of perturbations of propagated Schrödinger eigenfunctions
}

\author{
Yaiza Canzani, Dmitry Jakobson, and John Toth ${ }^{1}$
}

\begin{abstract}
Let $\left(M, g_{0}\right)$ be a compact Riemannian manifold of dimension $n$. Let $P_{0}(h) \stackrel{\text { def }}{=}$ $-h^{2} \Delta_{g}+V$ be the semiclassical Schrödinger operator for $h \in\left(0, h_{0}\right]$, and let $E$ be a regular value of its principal symbol. Write $\varphi_{h}$ for an $L^{2}$-normalized eigenfunction of $P_{0}(h)$ with eigenvalue $E(h) \in[E-o(1), E+o(1)]$. We consider a smooth family of metric perturbations $g_{u}$ of $g_{0}$ with $u$ in the product space $B^{k}(\varepsilon)=(-\varepsilon, \varepsilon)^{k} \subset \mathbb{R}^{k}$ satisfying the admissibility condition in Definition 1. For $P_{u}(h) \stackrel{\text { def }}{=}-h^{2} \Delta_{g_{u}}+V$ and small $|t|>0$, we define the
\end{abstract} propagated perturbed eigenfunctions

$$
\varphi_{h, t}^{(u) \stackrel{\text { def }}{=}} e^{-\frac{i}{h} t P_{u}(h)} \varphi_{h} .
$$

They appear in the mathematical description of the Loschmidt echo effect in physics.

Motivated by random wave conjectures in quantum chaos, we study the distribution of the real part of the perturbed eigenfunctions regarded as random variables

$$
\operatorname{Re}\left(\varphi_{h, t}^{(\cdot)}(x)\right): B^{k}(\varepsilon) \longrightarrow \mathbb{R}, \quad x \in M .
$$

In particular, under an admissibility condition on the metric when $(M, g)$ is chaotic, we compute the $h \rightarrow 0^{+}$asymptotics of the variance $\operatorname{Var}\left[\operatorname{Re}\left(\varphi_{h, t}^{(\cdot)}(x)\right)\right]$ and show that the odd moments vanish as $h \rightarrow 0^{+}$as long as $x$ is not on the generalized caustic set where $V(x)=E$.

Mathematics Subject Classification (2010). 35P20, 58J37, 58J40, 58J50, 58J51, 81Q15, $81 \mathrm{Q} 50$.

Keywords. Eigenfunctions, Schrödinger operators, Loschmidt echo, random wave conjecture, conformal and volume-preserving deformations.

\section{Introduction}

Let $\left(M, g_{0}\right)$ be a compact Riemannian manifold of dimension $n$ with Laplace operator

$$
\Delta_{g_{0}}=\delta_{g_{0}} d: C^{\infty}(M) \longrightarrow C^{\infty}(M),
$$

\footnotetext{
${ }^{1}$ Yaiza Canzani was supported by Schulich Fellowship. Dmitry Jakobson and John Toth were supported by NSERC, FQRNT and Dawson Fellowships.
} 
and let $V \in C^{\infty}(M)$ denote a smooth potential over $M$. For $h \in\left(0, h_{0}\right]$, consider the Schrödinger operator

$$
P_{0}(h) \stackrel{\text { def }}{=}-h^{2} \Delta_{g_{0}}+V,
$$

and let $E$ be a regular value of its principal symbol

$$
p_{0}(x, \xi) \stackrel{\text { def }}{=}|\xi|_{g_{0}(x)}^{2}+V(x)
$$

Write $\varphi_{h}$ for an $L^{2}$-normalized eigenfunction of $P(h)$ with eigenvalue contained in a shrinking interval centered at $E$; that is, $P_{0}(h) \varphi_{h}=E(h) \varphi_{h}$ and $E(h) \in$ $[E-o(1), E+o(1)]$.

Consider a smooth family of perturbations $g_{u}$ of the reference metric $g_{0}$ with $u$ in the product space $B^{k}(\varepsilon)=(-\varepsilon, \varepsilon)^{k} \subset \mathbb{R}^{k}$. The number of parameters $k \geq n$ is chosen sufficiently large (but finite) so that the admissibility condition on the perturbation $g_{u}$ in Definition 1 is satisfied. We introduce the associated perturbed Schrödinger operators

$$
P_{u}(h) \stackrel{\text { def }}{=}-h^{2} \Delta_{g_{u}}+V,
$$

with principal symbol

$$
p_{u}: T^{*} M \longrightarrow T^{*} M, \quad p_{u}(x, \xi) \stackrel{\text { def }}{=}|\xi|_{g_{u}}^{2}+V(x) .
$$

Fix $t \neq 0$ small, independent of $h$, and define the perturbed propagated eigenfunctions

$$
\varphi_{h, t}^{(u)} \stackrel{\text { def }}{=} e^{-\frac{i}{h} t P_{u}(h)} \varphi_{h}
$$

The perturbations satisfy $\varphi_{h, t}^{(u)}=\Phi_{h}^{(u)}(t)$ where $\Phi_{h}^{(u)}(t)$ denotes the solution at time $t$ of the Schrödinger equation

$$
\left\{\begin{array}{l}
\left(i h \frac{\partial}{\partial s}-P_{u}(h)\right) \Phi_{h}^{(u)}(s)=0, \\
\Phi_{h}^{(u)}(0)=\varphi_{h} .
\end{array}\right.
$$

The aim of this paper is to study the $h \rightarrow 0^{+}$asymptotics of the distribution of $\varphi_{h, t}^{(u)}$, where the latter are regarded as random variables in $u \in B^{k}(\varepsilon)$. Specifically, we compute the variance and all odd moments in the semiclassical limit $h \rightarrow 0^{+}$. To state our results, we need to define an admissibility condition on the metric perturbations. Here and throughout the rest of the manuscript we adopt the notation $\delta_{u_{\alpha}}=\left.\partial_{u_{\alpha}}\right|_{u=0}$. 
Definition 1 (admissibility condition). Let $g_{u}$ with $u \in B^{k}(\varepsilon)$ be a $C^{\infty}$ metric perturbation of a reference metric $g_{0}$. We say that $g_{u}$ is admissible at $x \in M$ if the following conditions are satisfied.

A) There exists an $n$-tuple of coordinates of $u, u^{\prime}=\left(u_{1}, \ldots, u_{n}\right)$, for which the Hessian matrices $d_{u^{\prime}} d_{\xi}\left(p_{u}(x, \xi)\right)$ are invertible for all $u \in B^{k}(\varepsilon)$ and all $\xi \in$ $T_{x}^{*} M$ with $(x, \xi) \in p_{0}^{-1}(E-c \varepsilon, E+c \varepsilon)$ where the constant $c=c(\varepsilon)>0$ is defined in (13).

B) There exists a parameter coordinate $u_{\alpha}$, a neighborhood $\mathcal{W}$ of $x$, and a function $a \in C^{\infty}(\mathcal{W}, \mathbb{R} \backslash\{0\})$ such that $\delta_{u_{\alpha}} g_{u}^{-1}(x)=a(x) g_{0}^{-1}(x)$ for $x \in \mathcal{W}$.

Remark 1. If $g_{u}$ is an admissible at $x$, then there exists a neighborhood $U \subset M$ of $x$ on which condition (A) holds.

We show in Section 5 that the admissibility condition in Definition 1 is satisfied by a large class of metric perturbations and we also give a geometric interpretation of the admissibility condition. The tangent space at $g_{0}$ to the space of all Riemannian metrics over $M$ can be decomposed into the direct sum of the space of symmetric 2-tensors with the fixed volume form $d v o l_{g_{0}}$, and the space of symmetric 2-tensors obtained by pointwise multiplication of $g_{0}$. We show that the notion of being admissible is intrinsically related to having $n=\operatorname{dim} M$ volume preserving directions in which the metric $g_{0}$ is perturbed (this is condition (A)) and to having one direction in which the metric can be conformally perturbed (this is condition (B)).

As a model example, suppose one wishes to perturb the flat metric $g_{0}$ on the 2-torus $\mathbb{T}^{2}$. Let $x_{0} \in \mathbb{T}^{2}$ and in a neighborhood $\mathcal{W}$ of $x_{0}$ consider any perturbation $g_{u}$ with $u=\left(u_{1}, u_{2}, u_{3}, u^{\prime \prime}\right) \in B^{k}(\varepsilon), k \geq 3$, of the form

$$
g_{u}^{-1}(x)=g_{0}^{-1}(x)+h_{\left(u_{1}, u_{2}, u_{3}\right)}(x)+h_{u^{\prime \prime}}(x), \quad x \in \mathcal{W},
$$

where

$$
\begin{aligned}
& h_{\left(u_{1}, u_{2}, u_{3}\right)}(x) \\
& \quad=u_{1}\left(\begin{array}{ll}
a_{1}(x) & b_{1}(x) \\
b_{1}(x) & -a_{1}(x)
\end{array}\right)+u_{2}\left(\begin{array}{cc}
a_{2}(x) & b_{2}(x) \\
b_{2}(x) & -a_{2}(x)
\end{array}\right)+u_{3}\left(\begin{array}{cc}
a_{3}(x) & 0 \\
0 & a_{3}(x)
\end{array}\right)
\end{aligned}
$$

and $h_{u^{\prime \prime}}$ is any symmetric 2-tensor depending on the left-out variables $u^{\prime \prime} \in B^{k-3}(\varepsilon)$ and higher powers of $u_{1}, u_{2}$ and $u_{3}$. The perturbation $g_{u}$ is admissible at $x_{0}$ provided $\varepsilon$ is small, $a_{2}, a_{2}, a_{3} \in C^{\infty}(\mathcal{W}), a_{3} \neq 0$ in $\mathcal{W}$, and the vector fields $\left(a_{1}\left(x_{0}\right), b_{1}\left(x_{0}\right)\right)$ and $\left(a_{2}\left(x_{0}\right), b_{2}\left(x_{0}\right)\right)$ are linearly independent. We remark that this admissibility condition is satisfied on open subsets in the space of all $C^{\infty}$ metric perturbations on $\mathbb{T}^{2}$.

We next describe the sense in which the eigenfunctions $\varphi_{h, t}^{(u)}$ are regarded as random variables in the deformation parameters $u \in B^{k}(\varepsilon)$. Consider a cut-off 
function $\chi \in C_{0}^{\infty}\left(B^{k}(\varepsilon) ;[0,1]\right)$ with $\chi(u)=1$ for $u \in B^{k}(\varepsilon / 2)$. We introduce the normalization constant

$$
c_{k}(\varepsilon) \stackrel{\text { def }}{=}\left(\int_{B^{k}(\varepsilon)} \chi^{2}(u) d u\right)^{-1},
$$

and define the probability measure $v$ on $B^{k}(\varepsilon)$ by

$$
d \nu(u) \stackrel{\text { def }}{=} c_{k}(\varepsilon) \chi^{2}(u) d u .
$$

The introduction of the cut-off function in the definition of the probability measure is to ensure that all the integrands we consider, regarded as functions of $u$, are compactly supported in the interior of the ball, $B^{k}(\varepsilon)$. This is crucial for the $h$-microlocal characterization of the variance in Proposition 5.

We view the real part of the perturbed eigenfunctions $\varphi_{h, t}^{(u)}$ defined in (4) as random variables

$$
\operatorname{Re}\left(\varphi_{h, t}^{(\cdot)}(x)\right): B^{k}(\varepsilon) \longrightarrow \mathbb{R}
$$

depending on the spatial parameters $x \in M$. Since one can study the distribution of a random variable such as $\operatorname{Re}\left(\varphi_{h, t}^{(\cdot)}(x)\right)$ by understanding its moments, we dedicate this paper to study the asymptotics of the variance $\operatorname{Var}\left[\operatorname{Re}\left(\varphi_{h, t}^{(\cdot)}(x)\right)\right]$ and of the odd moments $\mathbb{E}\left[\operatorname{Re}\left(\varphi_{h, t}^{(\cdot)}(x)\right)\right]^{p}$ in the semiclassical limit $\hbar \rightarrow 0^{+}$.

Remark 2. Throughout the paper, we write that a condition holds locally uniformly in a set $U$ whenever it holds uniformly on compact subsets of $U$.

Our first result holds for general Riemannian manifolds $\left(M, g_{0}\right)$.

Theorem 1. Let $\left(M, g_{0}\right)$ be a compact Riemannian manifold of dimension $n$ and let $E$ be a regular value of $p_{0}$. Suppose $g_{u}$ is a perturbation of $g_{0}$ with $u \in B^{k}(\varepsilon) \subset \mathbb{R}^{k}$ that is admissible at every $x \in M$. Fix a positive integer $\tilde{p} \in \mathbb{Z}^{+}$. Then, for $\varepsilon>0$ and $|t|>0$ sufficiently small, depending on $\left(M, g_{0}\right)$ and $\tilde{p}$, there is $h_{0}(t, \varepsilon)>0$ such that for $h \in\left(0, h_{0}(t, \varepsilon)\right]$ and $x \notin V^{-1}(E)$,

(1) There exists a constant $C>0$, independent of $h$, with

$$
\operatorname{Var}\left[\operatorname{Re}\left(\varphi_{h, t}^{(\cdot)}(x)\right)\right] \leq C .
$$

(2) For $p \in \mathbb{Z}^{+}$odd with $p \leq \tilde{p}$,

$$
\mathbb{E}\left[\operatorname{Re}\left(\varphi_{h, t}^{(\cdot)}(x)\right)\right]^{p}=\mathcal{O}\left(h^{\infty}\right) .
$$

These estimates are locally uniform for $x \notin V^{-1}(E)$. 
Remark 3. We note that if $g_{u}$ is admissible for all $x \in M$, we prove that as $h \rightarrow 0^{+}$,

$$
\int_{B^{k}(\varepsilon)}\left|\varphi_{h, t}^{(u)}(x)\right|^{2} d v(u)=\mathcal{O}(1),
$$

provided $\varepsilon>0$ and $|t|>0$ are sufficiently small. Moreover, this estimate is uniform in $x \in M$. However, the statement of the corresponding result for the variance in Theorem 1 (1) requires the asymptotic vanishing of the mean (the first moment) for which we require the condition that $x \notin V^{-1}(E)$ (see (8)).

The assumption $x \notin V^{-1}(E)$ in Theorem 1 is used in the integration by parts argument in (33) to estimate the odd moments. At present, we do not know whether the estimates for odd moments away from these generalized turning points extend uniformly to all $x \in M$. This assumption is vacuous in the homogeneous case $V=0$.

If the metric perturbation $g_{u}$ is admissible, there exist $c>0$ and an $n$-tuple of $u$ coordinates denoted by $u^{\prime}=\left(u_{1}, \ldots, u_{n}\right) \in B^{n}(\varepsilon)$ for which $\left|d_{u^{\prime}} d_{\xi} p_{\left(u^{\prime}, u^{\prime \prime}\right)}(x, \eta)\right| \neq$ 0 at $u=0$ provided $(x, \eta) \in p_{0}^{-1}(E-c \varepsilon, E+c \varepsilon)$. Using this, we show via an Implicit Function Theorem argument that one can locally parametrize $u^{\prime}$ as a smooth function of $(y, \eta) \in p_{0}^{-1}(E), u^{\prime}=u^{\prime}(y, \eta)$ (see (42)). We write $u^{\prime \prime} \in B^{k-n}(\varepsilon)$ for the omitted parameters and the dependence of $u^{\prime}(y, \eta)$ on $\left(u^{\prime \prime}, x\right)$ as parameters is understood. Furthermore, without loss of generality, we assume that the coordinates of $u$ are ordered so that $u=\left(u^{\prime}, u^{\prime \prime}\right)$.

Let $H_{p_{u}}$ be the Hamiltonian vector field of $p_{u} \in C^{\infty}\left(T^{*} M\right)$ and denote by

$$
G_{u}^{s}: S_{g_{u}}^{*} M \longrightarrow S_{g_{u}}^{*} M
$$

the bicharacteristic flow associated to $H_{p_{u}}$ at time $s$. In the case where the manifold $\left(M, g_{0}\right)$ has an ergodic geodesic flow

$$
G_{0}^{s}: S^{*} M \longrightarrow S^{*} M
$$

we get asymptotic results for the variance provided we consider quantum ergodic sequences of eigenfunctions (for a precise definition see (9)). We continue to write $\chi$ for the cut-off function in the definition of the probability measure and $c_{k}(\varepsilon)$ for the corresponding normalizing factor (5).

Theorem 2. Let $\left(M, g_{0}\right)$ be a compact Riemannian manifold of dimension $n$ and let $E$ be a regular value of $p_{0}$. Assume the geodesic flow on $p_{0}^{-1}(E)$ is ergodic and that $\left\{\varphi_{h}\right\}_{h \in\left(0, h_{0}\right]}$ is a quantum ergodic sequence of $L^{2}$-normalized eigenfunctions of $P_{0}(h)$. Suppose $g_{u}$ with $u \in B^{k}(\varepsilon)$ is a perturbation of $g_{0}$ that is admissible at $x \notin V^{-1}(E)$. Fix a positive integer $\tilde{p} \in \mathbb{Z}^{+}$. Then, for $|t|>0$ and $\varepsilon>0$ sufficiently small, depending on $\left(M, g_{0}\right)$ and $\tilde{p}$, 
(1) we have

$$
\lim _{h \rightarrow 0^{+}} \operatorname{Var}\left[\operatorname{Re}\left(\varphi_{h, t}^{(\cdot)}(x)\right)\right]=\int_{B^{k-n}(\varepsilon)} \beta_{t, x}^{k}\left(u^{\prime \prime}\right) d u^{\prime \prime},
$$

where

$$
\beta_{t, x}^{k}: B^{k-n}(\varepsilon) \longrightarrow \mathbb{R}
$$

is defined by

$$
\begin{aligned}
& \beta_{t, x}^{k}\left(u^{\prime \prime}\right) \\
& \stackrel{\text { def }}{=} \frac{c_{k}(\varepsilon)(1+\mathcal{O}(t))}{|t|^{n}\left|p_{0}^{-1}(E)\right|} \\
& \quad \times \int_{p_{0}^{-1}(E)} \frac{\left|\operatorname{det}\left(d_{x} \pi G_{\left(u^{\prime}(y, \eta), u^{\prime \prime}\right)}^{-t}(x, \eta)\right)\right|}{\left|\operatorname{det}\left(d_{u^{\prime}} d_{\xi} p_{\left(u^{\prime}(y, \eta), u^{\prime \prime}\right)}(x, \eta)\right)\right|} \chi^{2}\left(u^{\prime}(y, \eta), u^{\prime \prime}\right) d \omega_{E}(y, \eta)
\end{aligned}
$$

and $u^{\prime}=u^{\prime}(y, \eta)$ is defined in (42);

(2) for $p \in \mathbb{Z}^{+}$odd with $p \leq \tilde{p}$,

$$
\lim _{h \rightarrow 0^{+}} \mathbb{E}\left[\operatorname{Re}\left(\varphi_{h, t}^{(\cdot)}(x)\right)\right]^{p}=0 .
$$

1.1. Motivation. We proceed to describe two ideas that motivate our work. We first explain how the underlying ideas in our approach are motivated by the random wave conjecture. We then relate our results to the physics notion of Loschmidt echo.

Random wave conjecture. In 1977, M. Berry conjectured that the real and imaginary parts of the eigenfunctions $\varphi_{h}$ in the chaotic case resemble random waves; see [1]. It is also believed that the eigenfunctions $\varphi_{h}$ of quantum mixing systems behave locally as independent Gaussian variables as $h \rightarrow 0$; see for example the discussion in [9] and references therein. One of the common issues is to define a probability model where the random functions mimic the chaotic eigenfunctions. This is the role we give to the perturbations $\varphi_{h, t}^{(u)}$.

Loschmidt echo. A natural way of measuring the noise affecting a given system is the Loschmidt echo. The idea behind this concept is to measure the sensitivity of quantum evolution to perturbations, by propagating forward an initial state $\psi$ using the unperturbed Hamiltonian $p_{0}$, and propagating it back via the perturbed one $p_{u}$ after time $t$. Thus, the objects of interest in this case are the states $e^{\frac{i t}{h} P_{u}(h)} e^{-\frac{i t}{h} P_{0}(h)} \psi$ and the Loschmidt Echo, $M_{\mathrm{LE}}(t)$, is defined to be the return probability to the initial state:

$$
M_{\mathrm{LE}}(t)=\left|\left\langle e^{-\frac{i t}{h} P_{u}(h)} e^{\frac{i t}{h} P_{0}(h)} \psi, \psi\right\rangle\right|^{2} .
$$




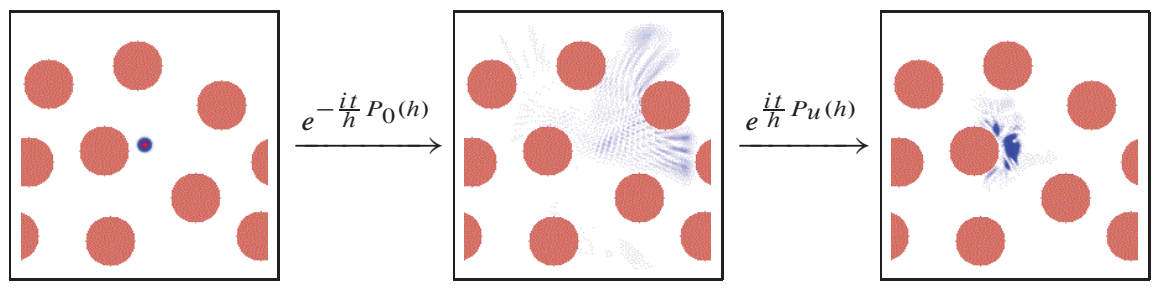

Figure 1. Illustration of the state of particle initially placed in the center of a square billiard with an irregular array of 10 circular scatterers with initial momentum pointing to the left [3].

We are interested in the case when the initial state $\psi$ is an eigenfunction, $\psi=\varphi_{h}$. In this simpler case $M_{\mathrm{LE}}(t)$ is called the survival probability [16] and we have

$$
e^{\frac{i t}{h} P_{u}(h)} e^{-\frac{i t}{h} P_{0}(h)} \varphi_{h}=e^{-\frac{i t E(h)}{h}} \varphi_{h, t}^{(u)} .
$$

To be precise, for an initial state $\varphi_{h}$ the Loschmidt Echo is simply

$$
M_{\mathrm{LE}}(t)=\left|\left\langle\varphi_{h, t}^{(u)}, \varphi_{h}\right\rangle\right|^{2}
$$

As the definition shows, the fidelity $M_{\mathrm{LE}}(t)$ can be interpreted as the decaying overlap between the evolution $\varphi_{h, t}^{(u)}$ and the unperturbed evolution $\varphi_{h}$; see [11], [10], and [13].

In recent work [8], Eswarathasan and Toth have proved related results for magnetic deformations of the Hamiltonian $p_{0}(x, \xi)=|\xi|_{g_{0}(x)}^{2}+V(x)$. We extend their upper bound results to large families of metric deformations. In addition, we characterize the asymptotic results in terms of variance and show that all odd moments are negligible. Although we do not have a rigorous argument at the moment, we hope that by further developing the methods of the present paper, we will be able to compute the higher even moments $\lim _{h \rightarrow 0^{+}} \mathbb{E}\left[\operatorname{Re}\left(\varphi_{h, t}^{(\cdot)}(x)\right)\right]^{2 p}$ for $p \geq 2$, and compare them with the Gaussian prediction of the random wave model. We plan to return to this question elsewhere.

1.2. Outline of the paper In Section 2 we introduce the background material and notation from semiclassical analysis that we shall use to prove our results. We first show that the perturbations are semiclassically localized in $p_{0}^{-1}(E)$ and then explain how to microlocally cut off the propagator $e^{-\frac{i t}{h} P_{u}(h)}$ to obtain a localized approximation of $\varphi_{h, t}^{(u)}$.

In Section 3, we study the odd moments of $\operatorname{Re}\left(\varphi_{h, t}^{(\cdot)}(x)\right)$. Provided the metric perturbation satisfies part (B) of the admissibility condition at $x_{*} \in M$, we prove in Lemma 4 that for fixed $\tilde{p} \in \mathbb{Z}^{+}$and $\ell, q \in \mathbb{Z}^{+}$with $1 \leq \ell \leq \tilde{p}$ and $2 q \leq \tilde{p}$,

$$
\int_{B^{k}(\varepsilon)}\left(\varphi_{h, t}^{(u)}(x)\right)^{\ell}\left|\varphi_{h, t}^{(u)}(x)\right|^{2 q} d \nu(u)=\mathcal{O}\left(h^{\infty}\right) \quad \text { as } h \rightarrow 0^{+},
$$


for $\varepsilon>0$ and $|t|>0$ sufficiently small depending on $\left(M, g_{0}\right)$ and $\tilde{p}$. The error is locally uniform in $x \in \mathcal{W} \cap\left(V^{-1}(E)\right)^{c}$ where $\mathcal{W}$ is the open neighborhood of $x_{*}$ given by part (B) of the admissibility condition.

Using Lemma 4 and the binomial expansion for $(\varphi+\bar{\varphi})^{p}=(2 \operatorname{Re} \varphi)^{p}$, we prove that for $p \in \mathbb{Z}^{+}$odd,

$$
\mathbb{E}\left[\operatorname{Re}\left(\varphi_{h, t}^{(\cdot)}(x)\right)\right]^{p}=\mathcal{O}\left(h^{\infty}\right),
$$

locally uniformly in $W \cap\left(V^{-1}(E)\right)^{c}$.

In Section 4 we study the variance of $\operatorname{Re}\left(\varphi_{h, t}^{(\cdot)}(x)\right)$. Provided the perturbation is admissible at $x \in\left(V^{-1}(E)\right)^{c}$, the case $p=1$ in (7) shows that our variables are semiclassically centered in the sense

$$
\mathbb{E}\left[\operatorname{Re}\left(\varphi_{h, t}^{(\cdot)}(x)\right)\right]=\mathcal{O}\left(h^{\infty}\right) .
$$

Therefore,

$$
\operatorname{Var}\left[\operatorname{Re}\left(\varphi_{h, t}^{(\cdot)}(x)\right)\right]=\int_{B^{k}(\varepsilon)}\left|\varphi_{h, t}^{(u)}(x)\right|^{2} d v(u)+\mathcal{O}\left(h^{\infty}\right) .
$$

It follows that studying the variance is equivalent to understanding the behavior of the right hand side in the previous equality. In Proposition 5 we compute the asymptotics of the RHS in (8) and consequently prove Theorems 1 and 2.

In Section 5 we show that there always exist large families of admissible perturbations. We show that the notion of admissibility is related to having sufficiently many volume preserving directions in which the metric tensor $g_{0}$ is perturbed (this is Condition A), and to having at least one direction in which $g_{0}$ is conformally perturbed (this is Condition B).

Remark 4. We note here that there is an easy consequence of Theorem 1 (see also Remark 3) that concerns restriction bounds of $\varphi_{h, t}^{(u)}$ to smooth submanifolds $H \subset M$ under the assumption that the family $g_{u}$ is admissible for all $x \in M$. Indeed, since our upper bounds are then uniform in $x \in M$ (see Remark 3), by integrating over $H$ and applying Fubini, one gets that for $h \in\left(0, h_{0}\right]$, there exist a constant $C=$ $C\left(H, h_{0}\right)>0$ with

$$
\int_{B^{k}(\varepsilon)} \int_{H}\left|\varphi_{h, t}^{(u)}(s)\right|^{2} d \sigma_{H}(s) d v(u) \leq C .
$$

By the Tschebyshev inequality, it then follows that for any sequence $\omega(h)=o(1)$ as $h \rightarrow 0^{+}$, there is a measurable $D(h) \subset B^{k}(\varepsilon)$ with $\lim _{h \rightarrow 0^{+}} \frac{|D(h)|}{\left|B^{k}(\varepsilon)\right|}=1$ such that for $u \in D(h)$,

$$
\int_{H}\left|\varphi_{h, t}^{(u)}(s)\right|^{2} d \sigma_{H}(s)=\mathcal{O}\left(|\omega(h)|^{-1}\right) .
$$

Therefore, the restriction bounds for most perturbed eigenfunctions are much smaller than the universal bounds for $\int_{H}\left|\varphi_{h, t}^{(0)}(s)\right|^{2} d \sigma_{H}(s)$ in [2], Theorem 3, and tend to be consistent with the ergodic case; see [5] and [15]. 
Acknowledgements. The authors would like to thank the referee for many helpful detailed comments on the manuscript and for pointing out an error in the previous version of the paper.

\section{Background and Notation}

In this section we introduce some background material on eigenfunction localization and semiclassically cut off propagators. Most of this is standard in semiclassical analysis, but we include it for the benefit of the reader. We refer to [17] for further details.

Let $M$ be a compact Riemannian manifold of dimension $n$. We work with the class of semiclassical symbols

$$
\begin{aligned}
& S_{\mathrm{cl}}^{m, k}\left(T^{*} M\right) \\
& \stackrel{\text { def }}{=}\left\{a \in C^{\infty}\left(T^{*} M \times\left(0, h_{0}\right]\right): a(x, \xi ; h) \sim_{h \rightarrow 0^{+}} h^{-m} \sum_{j=1}^{\infty} a_{j}(x, \xi) h^{j}\right. \\
& \text { with } \left.\left|\partial_{x}^{\alpha} \partial_{\xi}^{\beta} a_{j}(x, \xi)\right| \leq C_{\alpha, \beta}\left(1+|\xi|^{2}\right)^{\frac{k-|\beta|}{2}}\right\} .
\end{aligned}
$$

For $a \in S_{\mathrm{cl}}^{m, k}\left(T^{*} M\right)$, we consider the Schwartz kernel in $M \times M$ locally of the form

$$
O p_{h}(a)(x, y)=\frac{1}{(2 \pi h)^{n}} \int_{\mathbb{R}^{n}} e^{\frac{i}{h}\langle x-y, \xi\rangle} a(x, \xi ; h) d \xi,
$$

for $(x, y) \in U \times V$ where $U, V \subset \mathbb{R}^{n}$ are local coordinate charts. The corresponding space of pseudodifferential operators is defined to be

$$
\Psi_{\mathrm{cl}}^{m, k}(M) \stackrel{\text { def }}{=}\left\{O p_{h}(a): a \in S_{\mathrm{cl}}^{m, k}\left(T^{*} M\right)\right\} .
$$

Let $N$ be another compact n-dimensional Riemannian manifold. We also consider the class of Fourier integral operators $I_{\mathrm{cl}}^{m, k}(M \times N, \Gamma)$ with Schwartz kernels defined in the form

$$
F_{h}(x, y)=\frac{1}{(2 \pi h)^{n}} \int_{\mathbb{R}^{n}} e^{\frac{i}{h} \varphi(x, y, \xi)} a(x, y, \xi ; h) d \xi
$$

for $(x, y) \in U \times V$ where $U, V \subset \mathbb{R}^{n}$ are local coordinate charts and

$$
a \in C_{0}^{\infty}\left(U \times V \times \mathbb{R}^{n} \times\left(0, h_{0}\right]\right)
$$

with

$$
a(x, y, \xi ; h) \sim_{h \rightarrow 0^{+}} h^{-m} \sum_{j=1}^{\infty} a_{j}(x, y, \xi) h^{j}
$$


Here $\varphi$ denotes a non-degenerate phase function in the sense of Hörmander [4], Definition (2.3.10), and $\Gamma$ is an immersed Lagrangian submanifold of $T^{*} M \times T^{*} M$ with

$$
\Gamma=\left\{\left(x, d_{x} \varphi, y,-d_{y} \varphi\right): d_{\xi} \varphi(x, y, \xi)=0\right\} \subset T^{*} M \times T^{*} N .
$$

Finally, throughout the manuscript we say that a sequence of $L^{2}$-normalized eigenfunctions $\left\{\varphi_{h_{j}}\right\}_{j \geq 1}$ of $P_{0}\left(h_{j}\right)$ with $P_{0}\left(h_{j}\right) \varphi_{h_{j}}=E\left(h_{j}\right) \varphi_{h_{j}}$ and $E\left(h_{j}\right)=E+o(1)$ is quantum ergodic $(\mathrm{QE})$ if for any

$$
a(x, \xi, h) \sim \sum_{k=0}^{\infty} a_{k}(x, \xi) h^{k} \in S_{\mathrm{cl}}^{0,0}\left(T^{*} M \times\left[0, h_{0}\right)\right)
$$

we have

$$
\left\langle O p_{h_{j}}(a) \varphi_{h_{j}}, \varphi_{h_{j}}\right\rangle \underset{j \rightarrow \infty}{\longrightarrow} \int_{p_{0}^{-1}(E)} a_{0}(x, \xi) d \omega_{E}(x, \xi)
$$

where, $d \omega_{E}$ is normalized Liouville measure on $p_{0}^{-1}(E)$.

2.1. Eigenfunction localization. Fix $t \neq 0$ and let $E$ be a regular value of $p_{0}$. Then, for $u \in B^{k}(\varepsilon)$ we introduce the cut-off functions on $T^{*} M$

$$
\chi_{E}^{(u)}(x, \xi)=\chi_{0}\left(p_{0}\left(G_{u}^{-t}(x, \xi)\right)-E\right),
$$

where $\chi_{0} \in C_{0}^{\infty}([-\varepsilon, \varepsilon] ;[0,1])$ equal to 1 on $[-\varepsilon / 2, \varepsilon / 2]$. Consequently,

$$
\operatorname{supp} \chi_{E}^{(0)} \subset p_{0}^{-1}([E-\varepsilon, E+\varepsilon]) .
$$

Since $\chi_{E}^{(u)}(x, \xi)=\chi_{0}\left(p_{0}(x, \xi)-E+\mathcal{O}(|u|)\right)$, the support of $\chi_{E}^{(u)}$ remains localized near the hypersurface $p_{0}^{-1}(E)$ for all $u \in B^{k}(\varepsilon)$ (see (13) below for precise control) and that $\varphi_{h, t}^{(u)}$ is a normalized eigenfunction of the operator

$$
Q_{u}(h) \stackrel{\text { def }}{=} e^{-\frac{i}{h} t P_{u}(h)} P_{0}(h) e^{\frac{i}{h} t P_{u}(h)} \in \Psi_{\mathrm{cl}}^{0,2}(M)
$$

with eigenvalue $E(h)$. By Egorov's Theorem $Q_{u}(h)=O p_{h}\left(p_{0} \circ G_{u}^{-t}\right)+\mathcal{O}_{L^{2} \rightarrow L^{2}}(h)$, and since $E(h) \in[E-o(1), E+o(1)]$, it follows that $\left(Q_{u}(h)-E\right) \varphi_{h, t}^{(u)}=o(1)$. Using that $Q_{u}(h)$ is $h$-elliptic off $\left(p_{0} \circ G_{u}^{-t}\right)^{-1}(E)$, a parametric construction [17], Theorem 6.4, gives $\left\|\varphi_{h, t}^{(u)}-O p_{h}\left(\chi_{E}^{(u)}\right) \varphi_{h, t}^{(u)}\right\|_{L^{2}}=\mathcal{O}\left(h^{\infty}\right)$ and therefore $W F_{h}\left(\varphi_{h, t}^{(u)}\right) \subset$ $\left(p_{0} \circ G_{u}^{-t}\right)^{-1}(E)$. Fix $t \neq 0$ and $\varepsilon>0$ small. Given $x \in M$, there is a coordinate chart $U$ with $x \in U$ and such that $\pi\left(G_{u}^{-t}(x, \xi)\right) \subset U$ for $u \in B^{k}(\varepsilon)$. Then, since $p_{0}\left(G_{0}^{-t}(x, \xi)\right)=p_{0}(x, \xi)$, by Taylor expansion around $u=0$,

$$
p_{0}\left(G_{u}^{-t}(x, \xi)\right)=p_{0}(x, \xi)+R_{1}(x, \xi ; u, t)
$$

where

$$
\left|R_{1}(x, \xi, u ; t)\right| \leq \sqrt{k} \max _{u \in B^{k}(\varepsilon)}\left\|d_{u}\left(p_{0} \circ G_{u}^{-t}\right)(x, \xi)\right\||u| .
$$


Here, given a arbitrary matrix $A=\left(a_{i j}\right)$ we write

$$
\|A\| \stackrel{\text { def }}{=} \max _{i, j}\left|a_{i j}\right| .
$$

For $t \neq 0$ fixed, we define the constant

$$
c \stackrel{\text { def }}{=} k \max _{u \in B^{k}(\varepsilon)} \max _{\left(p_{0} \circ G_{u}^{-t}\right)^{-1}(E)}\left\|d_{u}\left(p_{0} \circ G_{u}^{-t}\right)(x, \xi)\right\| .
$$

It then follows from (12) that

$$
\left.\left(p_{0} \circ G_{u}^{-t}\right)^{-1}(E) \subset p_{0}^{-1}(E-c\|u\|, E+c\|u\|)\right)
$$

for $c>0$ in (13) and $u \in B^{k}(\varepsilon)$. Consequently, $W F_{h}\left(\varphi_{h, t}^{(u)}\right) \subset p_{0}^{-1}(E-c \varepsilon, E+c \varepsilon)$ and by a Sobolev lemma argument one can also prove $\left\|\varphi_{h, t}^{(u)}-O p_{h}\left(\chi_{E}^{(u)}\right) \varphi_{h, t}^{(u)}\right\|_{C^{k}}=$ $\mathcal{O}_{C^{k}}\left(h^{\infty}\right)$. It follows that

$$
\varphi_{h, t}^{(u)}=O p_{h}\left(\chi_{E}^{(u)}\right) \circ e^{-\frac{i t}{h} P_{u}(h)} \circ O p_{h}\left(\chi_{E}^{(0)}\right) \varphi_{h}+\mathcal{O}_{C^{k}}\left(h^{\infty}\right),
$$

and from (14) and (11), with $c>0$ in (13),

$$
\operatorname{supp} \chi_{E}^{(u)} \subset p_{0}^{-1}([E-\varepsilon-c\|u\|, E+\varepsilon+c\|u\|]) .
$$

2.2. Semiclassically cut off propagators. Motivated by the approximation (15), for $h \in\left(0, h_{0}\right], u \in B^{k}(\varepsilon)$ and $|t|>0$ small, we define the semiclassically cut off Fourier integral operators $W_{t, u}(h) \in I_{\mathrm{cl}}^{0,-\infty}\left(M \times M, \Gamma_{u, t}\right)$,

$$
W_{t, u}(h) \stackrel{\text { def }}{=} O p_{h}\left(\chi_{E}^{(u)}\right) \circ e^{-\frac{i t}{h} P_{u}(h)} \circ O p_{h}\left(\chi_{E}^{(0)}\right),
$$

with immersed Lagrangian,

$$
\Gamma_{u, t}=\left\{(x, \xi ; y, \eta):(x, \xi)=G_{u}^{-t}(y, \eta),(y, \eta) \in \operatorname{supp} \chi_{E}^{(0)}\right\} \subset T^{*} M \times T^{*} M .
$$

We note that since $G_{u}^{-t}$ is then a symplectomorphism that is close to the identity, there exists a local generating function $S(s, u, \xi ; x)$ with $\left(x, d_{x} S(s, u, \eta ; x)\right)=$ $G_{u}^{-t}\left(d_{\eta} S(s, u, \eta ; x, \eta), \eta\right)$ for $s$ close to $t$. It follows that

$$
\begin{gathered}
\Gamma_{u, t}=\left\{\left(x, d_{x} S(t, u, \eta ; x) ; d_{\eta} S(t, u, \eta ; x), \eta\right) \in \operatorname{supp} \chi_{E}^{(u)} \times \operatorname{supp} \chi_{E}^{(0)}\right\} \\
\subset T^{*} M \times T^{*} M .
\end{gathered}
$$

The generating function $S(s, u, \eta ; x)$ solves the Hamilton-Jacobi initial value problem

$$
\left\{\begin{array}{l}
\partial_{s} S(s, u, \eta ; x)+p_{u}\left(x, d_{x} S(s, u, \eta ; x)\right)=0, \\
S(0, u, \eta ; x)=\langle x, \eta\rangle,
\end{array}\right.
$$


and therefore, a Taylor expansion in $s$ around $s=0$ gives

$$
S(s, u, \eta ; x)=\langle x, \eta\rangle-s p_{u}(x, \eta)+\mathcal{O}\left(s^{2}\right) .
$$

Given local coordinate charts $U, V \subset \mathbb{R}^{n}$ consider the local phase function $\varphi_{t} \in$ $C^{\infty}\left(V \times B^{k}(\varepsilon) \times \mathbb{R}^{n}\right)$,

$$
\varphi_{t}(y, u, \xi ; x) \stackrel{\text { def }}{=} S(t, u, \xi ; x)-\langle y, \xi\rangle
$$

The Schwartz kernel of $W_{t, u}(h)$ is locally of the form

$$
W_{t, u}(h)(x, y)=\frac{1}{(2 \pi h)^{n}} \int_{\mathbb{R}^{n}} e^{\frac{i}{h} \varphi_{t}(y, u, \xi ; x)} a_{t}(u, y, \xi ; x, h) d \xi+K_{x}(y, u),
$$

where $\left|\partial_{x}^{\alpha} \partial_{y}^{\beta} K_{x}(y, u)\right|=\mathcal{O}_{\alpha, \beta}\left(h^{\infty}\right)$ uniformly for $(y, x, u) \in V \times U \times B^{k}(\varepsilon)$.

The amplitude $a_{t}(u, y, \xi ; x, h) \sim \sum_{j=0}^{\infty} a_{t, j}(u, y, \xi ; x) h^{j}$ with

$$
a_{t, j}(u, \cdot, \cdot ; \cdot) \in C^{\infty}\left(B^{k}(\varepsilon), C_{0}^{\infty}\left(V \times \mathbb{R}^{n} \times U\right)\right) .
$$

From (14) it is clear that the support of $\chi_{E}^{(u)}$ remains localized near the hypersurface $p_{0}^{-1}(E)$ for all $u \in B^{k}(\varepsilon)$; indeed, with the constant $c>0$ in (13),

$$
\begin{aligned}
\operatorname{supp}\left(a_{t}(u, \cdot, \cdot ; \cdot, h)\right) \subset\{ & (y, \xi, x) \in T^{*} U \times V: \\
& (y, \xi) \in \operatorname{supp} \chi_{E}^{(0)} \subset p_{0}^{-1}(E-\varepsilon, E+\varepsilon), \\
& \left.y=d_{\xi} S(t, u, \xi ; x)=x+\mathcal{O}_{u}(t)\right\} .
\end{aligned}
$$

We note that since $t \neq 0$ is a fixed small parameter and $u \in B^{k}(\varepsilon)$ with $\varepsilon>0$ small, it follows from (22) that the amplitudes $a_{t, j}(u, \cdot, \cdot ; \cdot)$ are supported near the set $\left\{(y, \xi, y) \in T^{*} U \times U\right\}$. Similarly, the Lagrangian manifolds $\Gamma_{t, u} \subset T^{*} M \times T^{*} M$ are localized near the diagonal $\Delta_{T^{*} M \times T^{*} M}=\left\{(x, \xi ; x, \xi) \in T^{*} M \times T^{*} M\right\}$.

\section{Odd moments}

The purpose of this section is to show that provided the metric $g_{0}$ is conformally deformed in at least one direction, its odd moments are negligible for general geodesic flows. Throughout this section we continue to assume that $\left(M, g_{0}\right)$ is a compact Riemannian manifold and $E$ is a regular value of $p_{0}$. We prove

Proposition 3. Let $g_{u}$ with $u \in B^{k}(\varepsilon)$ be a perturbation of $g_{0}$ that satisfies part (B) of the admissibility condition at $x_{*} \in M$. Fix $\tilde{p} \in \mathbb{Z}^{+}$and suppose $p \in \mathbb{Z}^{+}$is odd with $p \leq \tilde{p}$. Then, for $\varepsilon>0$ and $|t|>0$ sufficiently small depending on $\left(M, g_{0}\right)$ and $\tilde{p}$,

$$
\mathbb{E}\left[\operatorname{Re}\left(\varphi_{h, t}^{(\cdot)}(x)\right)\right]^{p}=\mathcal{O}\left(h^{\infty}\right) \quad \text { as } h \rightarrow 0^{+},
$$

locally uniformly for $x \in \mathcal{W} \cap\left(V^{-1}(E)\right)^{c}$. Here $\mathcal{W}$ is the open neighborhood of $x_{*}$ given by part (B) of the admissibility condition. 
Proof. Given $p \leq \tilde{p}$ odd,

$$
\mathbb{E}\left[\operatorname{Re}\left(\varphi_{h, t}^{(\cdot)}(x)\right)\right]^{p}=\int_{B^{k}(\varepsilon)}\left(\operatorname{Re}\left(\varphi_{h, t}^{(u)}(x)\right)\right)^{p} d \nu(u),
$$

and for any complex $\varphi$ the binomial expansion of $(\varphi+\bar{\varphi})^{p}=(2 \operatorname{Re} \varphi)^{p}$ for $p$ odd gives

$$
(\operatorname{Re} \varphi)^{p}=\frac{1}{2^{p}} \sum_{0 \leq j<\frac{p}{2}}\left(\begin{array}{l}
p \\
j
\end{array}\right) \varphi^{p-2 j}|\varphi|^{2 j}+\frac{1}{2^{p}} \sum_{\frac{p}{2}<j \leq p}\left(\begin{array}{l}
p \\
j
\end{array}\right) \bar{\varphi}^{2 j-p}|\varphi|^{2(p-j)} .
$$

Therefore, to prove Proposition 3, it suffices to show that

$$
\int_{B^{k}(\varepsilon)}\left(\varphi_{h, t}^{(u)}(x)\right)^{\ell}\left|\varphi_{h, t}^{(u)}(x)\right|^{2 q} d \nu(u)=\mathcal{O}\left(h^{\infty}\right), \quad 1 \leq \ell \leq \tilde{p}, 2 q \leq \tilde{p},
$$

locally uniformly in $x \in \mathcal{W} \cap\left(V^{-1}(E)\right)^{c}$ as $h \rightarrow 0^{+}$.

Since the proof of (25) is somewhat technical, we prove it separately as Lemma 4. Combining (25) with the binomial expansion (24) completes the proof.

We have reduced the proof of Proposition 3 to establishing the following Lemma.

Lemma 4. Let $g_{u}$ with $u \in B^{k}(\varepsilon)$ be a perturbation of $g_{0}$ that satisfies part (B) of the admissibility condition at $x_{*} \in M$. Fix $\tilde{p} \in \mathbb{Z}^{+}$and suppose $\ell, q \in \mathbb{Z}^{+}$with $1 \leq \ell \leq \tilde{p}$ and $2 q \leq \tilde{p}$. Then, for $\varepsilon>0$ and $|t|>0$ sufficiently small depending on $\left(M, g_{0}\right)$ and $\tilde{p}$,

$$
\int_{B^{k}(\varepsilon)}\left(\varphi_{h, t}^{(u)}(x)\right)^{\ell}\left|\varphi_{h, t}^{(u)}(x)\right|^{2 q} d v(u)=\mathcal{O}\left(h^{\infty}\right) \quad \text { as } h \rightarrow 0^{+},
$$

locally uniformly for $x \in \mathcal{W} \cap\left(V^{-1}(E)\right)^{c}$. Here $\mathbb{W}$ is the open neighborhood of $x_{*}$ given by part (B) of the admissibility condition.

Proof. We identify the product manifold $M^{(\ell+2 q)}$ with $M^{(\ell)} \times M^{(q)} \times M^{(q)}$ and write

$$
\left(\tilde{y}, \tilde{z}, \tilde{z}^{\prime}\right) \stackrel{\text { def }}{=}\left(y^{(1)}, \ldots, y^{(\ell)}, z^{(1)}, \ldots, z^{(q)}, z^{\prime(1)}, \ldots, z^{\prime(q)}\right) \in V^{(\ell+2 q)}
$$

for the local coordinates.

By assumption, there exists $a \in C^{\infty}(M)$ so that $\delta_{u_{\alpha}} g_{u}^{-1}(x)=a(x) g_{0}^{-1}(x)$ with $a(x) \neq 0$ for all $x \in \mathcal{W}$. Let us continue to write $\chi$ for the cut-off function appearing in the definition of the probability measure $\nu$, and $c_{k}(\varepsilon)$ for the corresponding normalizing factor in (5). Since from $(15), \varphi_{h, t}^{(u)}(x)=\left[W_{t, u}(h) \varphi_{h}\right](x)+\mathcal{O}\left(h^{\infty}\right)$, 
writing $W_{t, u}(x, y)$ for the kernel of $W_{t, u}$ we get

$$
\begin{aligned}
& \int_{B^{k}(\varepsilon)}\left(\varphi_{h, t}^{(u)}(x)\right)^{\ell}\left|\varphi_{h, t}^{(u)}(x)\right|^{2 q} d \nu(u) \\
& =\int_{B^{k}(\varepsilon)}\left(\left[W_{t, u}(h) \varphi_{h}\right](x)\right)^{\ell}\left|\left[W_{t, u}(h) \varphi_{h}\right](x)\right|^{2 q} d v(u)+\mathcal{O}\left(h^{\infty}\right) \\
& =c_{k}(\varepsilon) \int_{B^{k}(\varepsilon)} \int_{M^{\ell+2 q}} B_{t, u}^{[\ell, q]}\left(\tilde{y}, \tilde{z}, \tilde{z}^{\prime} ; x, h\right) \varphi_{h}\left(y^{(i)}\right) \varphi_{h}\left(z^{(j)}\right) \overline{\varphi_{h}\left(z^{\prime(j)}\right)} \\
& \times \chi^{2}(u) d \tilde{y} d \tilde{z} d \tilde{z}^{\prime} d u+\mathcal{O}\left(h^{\infty}\right),
\end{aligned}
$$

where $B_{t, u}^{[\ell, q]} \in C^{\infty}\left(V^{(\ell+2 q)} \times U \times\left[0, h_{0}\right)\right)$ is defined by the formula

$$
B_{t, u}^{[\ell, q]}\left(\tilde{y}, \tilde{z}, \tilde{z}^{\prime} ; x, h\right) \stackrel{\text { def }}{=} \prod_{\substack{1 \leq i \leq \ell \\ 1 \leq j \leq q}} W_{t, u}\left(x, y^{(i)}\right) W_{t, u}\left(x, z^{(j)}\right) \overline{W_{t, u}\left(x, z^{\prime(j)}\right)} .
$$

From (21) we deduce the kernel expansion

$$
\begin{aligned}
& B_{t, u}^{[\ell, q]}\left(\tilde{y}, \tilde{z}, \tilde{z}^{\prime} ; x, h\right) \\
& =\frac{1}{(2 \pi h)^{n(2 q+\ell)}} \int_{\mathbb{R}^{n q}} \int_{\mathbb{R}^{n q}} \int_{\mathbb{R}^{n \ell}} e^{\frac{i}{h} \Phi_{t}^{[\ell, q]}\left(\tilde{y}, \tilde{z}, \tilde{z}^{\prime}, u, \tilde{\xi}, \tilde{\eta}, \tilde{\eta}^{\prime} ; x\right)} \times c_{t}^{[\ell, q]}\left(u, \tilde{y}, \tilde{z}, \tilde{z}^{\prime}, \tilde{\xi}, \tilde{\eta}, \tilde{\eta}^{\prime} ; x, h\right) d \tilde{\xi} d \tilde{\eta} d \tilde{\eta}^{\prime} \\
& \quad+K_{x}\left(\tilde{y}, \tilde{z}, \tilde{z}^{\prime}, u\right),
\end{aligned}
$$

for $\Phi_{t}^{[\ell, q]}, c^{[\ell, q]}$ and $K_{x}$ as follows.

(1) The phase function $\Phi_{t}^{[\ell, q]}$ is defined by

$$
\begin{aligned}
\Phi_{t}^{[\ell, q]}\left(\tilde{y}, \tilde{z}, \tilde{z}^{\prime}, u, \tilde{\xi}, \tilde{\eta}, \tilde{\eta}^{\prime} ; x\right) \\
\quad \stackrel{\text { def }}{=} \sum_{j=1}^{\ell} \varphi_{t}\left(y^{(j)}, u, \xi^{(j)} ; x\right)+\sum_{j=1}^{q} \varphi_{t}\left(z^{(j)}, u, \eta^{(j)} ; x\right)-\varphi_{t}\left(z^{(j)}, u, \eta^{(j)} ; x\right),
\end{aligned}
$$

where $\varphi_{t}$ is given in (20).

(2) The amplitude $c_{t}^{[\ell, q]}$ satisfies

$$
c_{t}^{[\ell, q]}\left(u, \tilde{y}, \tilde{z}, \tilde{z}^{\prime}, \tilde{\xi}, \tilde{\eta}, \tilde{\eta}^{\prime} ; x, h\right) \sim_{h \rightarrow 0^{+}} \sum_{j=0}^{\infty} c_{t, j}^{[\ell, q]}\left(u, \tilde{y}, \tilde{z}, \tilde{z}^{\prime}, \tilde{\xi}, \tilde{\eta}, \tilde{\eta}^{\prime} ; x\right) h^{j}
$$


with $c_{t, j}^{[\ell, q]}(u, \cdot, \cdot ; \cdot) \in C^{\infty}\left(B^{k}(\varepsilon), C_{0}^{\infty}\left(V^{(\ell+2 q)} \times \mathbb{R}^{n(\ell+2 q)} \times U\right)\right)$ for $U, V \subset \mathbb{R}^{n}$ local coordinate charts as in (20). Moreover,

$$
\begin{aligned}
& \operatorname{supp}\left(c_{t}^{[\ell, q]}\left(u, \tilde{y}, \tilde{z}, \tilde{z}^{\prime}, \cdot ; x, h\right)\right) \\
& \subset\left\{\left(\tilde{\xi}, \tilde{\eta}, \tilde{\eta}^{\prime}\right):\left(x, \xi^{(i)}\right),\left(x, \eta^{(j)}\right),\left(x, \eta^{\prime(j)}\right) \in p_{0}^{-1}(E-c \varepsilon, E+c \varepsilon),\right. \\
& \quad x \in U, i \leq \ell, j \leq q\} \\
& \subset \mathbb{R}^{n(\ell+2 q)} .
\end{aligned}
$$

(3) The residual operator $K_{x}$ satisfies

$$
\left|\partial_{x}^{\alpha} \partial_{\left(\tilde{y}, \tilde{z}, \tilde{z}^{\prime}\right)}^{\beta} K_{x}\left(\tilde{y}, \tilde{z}, \tilde{z}^{\prime}, u\right)\right|=\mathcal{O}_{\alpha, \beta}\left(h^{\infty}\right)
$$

locally uniformly in $\left(\tilde{y}, \tilde{z}, \tilde{z}^{\prime}, u\right) \in V^{(\ell+2 q)} \times B^{k}(\varepsilon)$.

Claim. For $\varepsilon>0$ and $|t|>0$ sufficiently small, there exists $C=C\left(t, \varepsilon, E, g_{0}\right)>0$ such that for $\left(\tilde{\xi}, \tilde{\eta}, \tilde{\eta}^{\prime}\right) \in \operatorname{supp}\left(c_{t}^{[\ell, q]}\left(u, \tilde{y}, \tilde{z}, \tilde{z}^{\prime}, \cdot ; x, h\right)\right)$,

$$
\left|\partial_{u_{\alpha}} \Phi_{t}^{[\ell, q]}\left(\tilde{y}, \tilde{z}, \tilde{z}^{\prime}, u, \tilde{\xi}, \tilde{\xi}^{\prime}, \tilde{\eta}, \tilde{\eta}^{\prime} ; x\right)\right| \geq C>0
$$

where this bound holds locally uniformly for $\left(\tilde{y}, \tilde{z}, \tilde{z}^{\prime}\right) \in M^{(\ell+2 q)}, u \in B^{k}(\varepsilon)$, and $x \in \mathcal{W} \cap\left(V^{-1}(E)\right)^{c}$.

To prove this claim we first observe that since $\delta_{u_{\alpha}} g_{u}^{-1}(x)=a(x) g_{0}^{-1}(x)$ for $x \in \mathcal{W}$,

$$
\delta_{u_{\alpha}} p_{u}(x, \xi)=a(x)|\xi|_{g_{0}(x)}^{2}
$$

Also, from the Taylor expansion of the generating function (19) around $s=0$, together with (20), we know that for $x \in \mathcal{W}$

$$
\varphi_{t}(y, u, \eta ; x)=\langle x-y, \eta\rangle-t p_{u}(x, \eta)+\mathcal{O}\left(t^{2}\right),
$$

where in (32), the error $\mathcal{O}\left(t^{2}\right)$ depends on $\tilde{p}$. Combining (28) with (32) and (31), for $x \in \mathcal{W}$ we get

$$
\begin{aligned}
& \partial_{u_{\alpha}} \Phi_{t}^{[\ell, q]}\left(\tilde{y}, \tilde{z}, \tilde{z}^{\prime}, u, \tilde{\xi}, \tilde{\eta}, \tilde{\eta}^{\prime} ; x\right)= \\
& =-\operatorname{ta}(x)\left(\sum_{i=1}^{\ell}\left|\xi^{(i)}\right|_{g_{0}(x)}^{2}+\sum_{j=1}^{q}\left|\eta^{(j)}\right|_{g_{0}(x)}^{2}-\sum_{j=1}^{q}\left|\eta^{\prime(j)}\right|_{g_{0}(x)}^{2}+\mathcal{O}(|u|)\right) \\
& \quad+\mathcal{O}\left(t^{2}\right) .
\end{aligned}
$$

From the support conditions on the amplitude $c_{t}^{[l, q]}$ in (29), we have that

$$
\begin{aligned}
& \left|\xi^{(j)}\right|_{g_{0}(x)}^{2}+V(x)=E+\mathcal{O}(\varepsilon), \\
& \left|\eta^{(j)}\right|_{g_{0}(x)}^{2}+V(x)=E+\mathcal{O}(\varepsilon),
\end{aligned}
$$


and

$$
\left|\eta^{\prime(j)}\right|_{g_{0}(x)}^{2}+V(x)=E+\mathcal{O}(\varepsilon),
$$

for $i \leq \ell$ and $j \leq q$. Therefore, for $x \in \mathcal{W}$,

$$
\begin{aligned}
& \partial_{u_{\alpha}} \Phi_{t}^{[\ell, q]}\left(\tilde{y}, \tilde{z}, \tilde{z}^{\prime}, u, \tilde{\xi}, \tilde{\eta}, \tilde{\eta}^{\prime} ; x\right) \\
& \quad=-t a(x)(\ell(E-V(x))+(\ell+2 q+1) \mathcal{O}(\varepsilon))+\mathcal{O}\left(t^{2}\right),
\end{aligned}
$$

and so

$$
\begin{aligned}
& \quad\left|\partial_{u_{\alpha}} \Phi_{t}^{[\ell, q]}\left(\tilde{y}, \tilde{z}, \tilde{z}^{\prime}, u, \tilde{\xi}, \tilde{\xi}^{\prime}, \tilde{\eta}, \tilde{\eta}^{\prime} ; x\right)\right| \\
& \quad \geq|\operatorname{ta}(x)| \cdot|\ell(E-V(x))+2 \tilde{p} \mathcal{O}(\varepsilon)|+\mathcal{O}\left(t^{2}\right),
\end{aligned}
$$

uniformly in all variables. Given a compact subset $K \subset \mathcal{W} \cap\left(V^{-1}(E)\right)^{c}$, for $x \in K$, one has $|V(x)-E| \geq \frac{1}{C_{0}}>0$ for some constant $C_{0}>0$. Since $a(x) \neq 0$ for all $x \in \mathcal{W}$, from (34) it follows that for such a given compact set $K$ and number of odd moments $\tilde{p} \in \mathbb{Z}^{+}$, we can choose $\varepsilon>0$ and $t \neq 0$ sufficiently small (depending on $\tilde{p}$ and $K$ ) so that the RHS of (34) is uniformly bounded away from zero. We conclude that the claim in (30) holds for $x \in \mathcal{W} \cap\left(V^{-1}(E)\right)^{c}$.

We then use the operator $\left(\frac{h}{i \partial_{u_{\alpha}} \Phi_{t}^{[\ell, q]}}\right) \frac{\partial}{\partial u_{\alpha}}$, to repeatedly integrate by parts in (26) and obtain

$$
B_{t, u}^{[\ell, q]}(h)\left(\tilde{y}, \tilde{z}, \tilde{z}^{\prime} ; x, h\right)=\mathcal{O}\left(h^{\infty}\right)
$$

locally uniformly for $\left(\tilde{y}, \tilde{z}, \tilde{z}^{\prime}\right) \in M^{(\ell+2 q)}, u \in B^{k}(\varepsilon)$ and $x \in \mathcal{W} \cap\left(V^{-1}(E)\right)^{c}$. We note that there are no boundary term contributions arising from the integration by parts since $\chi(u)=0$ for $u \in \partial B^{k}(\varepsilon)$. From (26) it follows that

$$
\int_{B^{k}(\varepsilon)}\left(\varphi_{h, t}^{(u)}(x)\right)^{\ell}\left|\varphi_{h, t}^{(u)}(x)\right|^{2 q} d \nu(u)=\mathcal{O}\left(h^{\infty}\right),
$$

locally uniformly in $x \in \mathcal{W} \cap\left(V^{-1}(E)\right)^{c}$.

\section{Variance}

As explained in the Introduction (see (8)), provided the perturbation is admissible at $x \in\left(V^{-1}(E)\right)^{c}$, the case $p=1$ in Proposition 3 shows that our random variables are semiclassically centered in the sense

$$
\mathbb{E}\left[\operatorname{Re}\left(\varphi_{h, t}^{(\cdot)}(x)\right)\right]=\mathcal{O}\left(h^{\infty}\right) .
$$

Therefore,

$$
\operatorname{Var}\left[\operatorname{Re}\left(\varphi_{h, t}^{(\cdot)}(x)\right)\right]=\int_{B^{k}(\varepsilon)}\left|\varphi_{h, t}^{(u)}(x)\right|^{2} d \nu(u)+\mathcal{O}\left(h^{\infty}\right)
$$


It then follows that studying the variance is equivalent to understanding the behavior of the right hand side in the previous equality. We compute the asymptotics of the RHS in the next Proposition.

Proposition 5. Let $g_{u}$ be admissible at $x_{*} \in M$ and let $U$ be the neighborhood of $x_{*}$ given in Remark 1. For $\varepsilon>0$ and $|t|>0$ sufficiently small, there exist a choice of coordinates $u^{\prime \prime} \in B^{k-n}(\varepsilon)$ and corresponding operators $A_{t, x, u^{\prime \prime}}(h) \in \Psi_{\mathrm{cl}}^{0,-\infty}(M)$ defined for all $\left(x, u^{\prime \prime}\right) \in U \times B^{k-n}(\varepsilon)$, such that

$$
\int_{B^{k}(\varepsilon)}\left|\varphi_{h, t}^{(u)}(x)\right|^{2} d \nu(u)=c_{k}(\varepsilon) \int_{B^{k-n}(\varepsilon)}\left\langle A_{t, x, u^{\prime \prime}}(h) \varphi_{\hbar}, \varphi_{\hbar}\right\rangle_{L^{2}(M)} d u^{\prime \prime}+\mathcal{O}\left(h^{\infty}\right) .
$$

Proof. By Remark 1, we choose $U \subset M$ to be an open neighborhood of $x_{*}$ so that the admissibility condition (A) holds on $U$. That is, given the constant $c>0$ in (13) and some subset of $n$ coordinates of $u$, which we denote $u^{\prime} \in B^{n}(\varepsilon)$, so that the matrix

$$
\begin{aligned}
& d_{u^{\prime}} d_{\xi}\left(p_{u}(x, \xi)\right) \text { is invertible for }(x, \xi) \in p_{0}^{-1}(E-c \varepsilon, E+c \varepsilon), \\
& (x, u) \in U \times B^{k}(\varepsilon) .
\end{aligned}
$$

We write $u^{\prime \prime} \in B^{k-n}(\varepsilon)$ for the omitted variables and assume that the coordinates of $u$ are ordered so that $u=\left(u^{\prime}, u^{\prime \prime}\right)$.

Write $W_{t, u}(h)(x, y)$ for the Schwartz kernel of $W_{t, u}(h)$. Then, for $u^{\prime \prime} \in B^{k-n}(\varepsilon)$ and $x \in \mathcal{U}$, we define a new family of operators

$$
\widehat{W}_{t, x, u^{\prime \prime}}(h): C^{\infty}(M) \longrightarrow C_{0}^{\infty}\left(B^{n}(\varepsilon)\right),
$$

with Schwartz kernels

$$
\widehat{W}_{t, x, u^{\prime \prime}}(h)\left(u^{\prime}, y\right) \stackrel{\text { def }}{=} \chi(u) \cdot W_{t, u}(h)(x, y), \quad u=\left(u^{\prime}, u^{\prime \prime}\right) \in B^{k}(\varepsilon),
$$

where we continue to write $\chi$ for the cut-off function appearing in the definition of the probability measure $v$ in (5). By (15),

$$
\chi(u) \varphi_{h, t}^{(u)}(x)=\chi(u)\left[W_{t, u}(h) \varphi_{h}\right](x)+\mathcal{O}\left(h^{\infty}\right)=\left[\widehat{W}_{t, x, u^{\prime \prime}}(h) \varphi_{h}\right]\left(u^{\prime}\right)+\mathcal{O}\left(h^{\infty}\right),
$$

and so,

$$
\begin{aligned}
\int_{B^{k}(\varepsilon)}\left|\varphi_{h, t}^{(u)}(x)\right|^{2} d v(u) \\
=c_{k}(\varepsilon) \int_{B^{k}(\varepsilon)}\left|\widehat{W}_{t, x, u^{\prime \prime}}(h) \varphi_{\hbar}\left(u^{\prime}\right)\right|^{2} d u+\mathcal{O}\left(h^{\infty}\right) \\
=c_{k}(\varepsilon) \int_{B^{k-n}(\varepsilon)}\left\langle\widehat{W}_{t, x, u^{\prime \prime}}(h) \varphi_{\hbar}, \widehat{W}_{t, x, u^{\prime \prime}}(h) \varphi_{\hbar}\right\rangle_{L^{2}\left(B^{n}(\varepsilon)\right)} d u^{\prime \prime}+\mathcal{O}\left(h^{\infty}\right) .
\end{aligned}
$$


From (21), the Schwartz kernel of $\widehat{W}_{t, x, u^{\prime \prime}}(h)$ is given by

$$
\begin{aligned}
& \widehat{W}_{t, x, u^{\prime \prime}}(h)\left(u^{\prime}, y\right) \\
& \quad=\frac{1}{(2 \pi h)^{n}} \int_{\mathbb{R}^{n}} e^{\frac{i}{h} \varphi_{t}\left(y, u^{\prime}, u^{\prime \prime}, \xi ; x\right)} a_{t}(u, y, \xi ; x, h) \chi(u) d \xi+K_{x}(y, u),
\end{aligned}
$$

where $\left|\partial_{x}^{\alpha} \partial_{y}^{\beta} K_{x}(y, u)\right|=\mathcal{O}_{\alpha, \beta}\left(h^{\infty}\right)$ uniformly in $(x, y, u) \in U \times V \times B^{k}(\varepsilon)$ for $\varepsilon>0$ small, where $U, V \subset \mathbb{R}^{n}$ are local coordinate charts with $U \subset \mathcal{U}$. The amplitude

$$
a_{t}(u, y, \xi ; x, h) \sim \sum_{j=0}^{\infty} a_{j}(u, y, \xi ; x) h^{j}
$$

with

$$
a_{j}(u, \cdot, \cdot ; \cdot) \in C^{\infty}\left(B^{k}(\varepsilon), C_{0}^{\infty}\left(V \times \mathbb{R}^{n} \times U\right)\right) .
$$

Moreover, we recall from (22) that

$$
\begin{array}{r}
\operatorname{supp}\left(a_{t}(u, \cdot, \cdot ; \cdot, h)\right) \subset\left\{(y, \xi, x) \in T^{*} U \times V:(x, \xi) \in p_{0}^{-1}(E-c \varepsilon, E+c \varepsilon),\right. \\
\left.y=d_{\xi} S(t, u, \xi ; x)=x+\mathcal{O}_{u}(t)\right\} .
\end{array}
$$

By the same argument presented in [8], Proposition 4.1, it can be shown that for $x \in U, \varepsilon>0$ and $|t| \neq 0$ small enough, $\widehat{W}_{t, x, u^{\prime \prime}}(h) \in I_{\mathrm{cl}}^{0,-\infty}\left(M \times B^{n}(\varepsilon) ; \Gamma_{t, x, u^{\prime \prime}}\right)$ with

$$
\begin{aligned}
& \Gamma_{t, x, u^{\prime \prime}} \\
& \left.\quad \stackrel{\text { def }}{=}\left\{\left(u^{\prime}, d_{u^{\prime}} S(t, u, \eta ; x)\right), d_{\eta} S(t, u, \eta ; x), \eta\right):\left(d_{\eta} S(t, u, \eta ; x), \eta\right) \in \operatorname{supp} \chi_{E}^{(0)}\right\} \\
& \quad \subset T^{*} B^{n}(\varepsilon) \times T^{*} M .
\end{aligned}
$$

where $u \stackrel{\text { def }}{=}\left(u^{\prime}, u^{\prime \prime}\right) \in B^{k}(\varepsilon)$ for $\varepsilon$ small, and $x \in U$. It remains to show that $\Gamma_{t, x, u^{\prime \prime}}$ is a canonical graph.

We recall that if $\left(d_{\eta} S(t, u, \eta ; x), \eta\right) \in \operatorname{supp} \chi_{E}^{(0)}$ for $u \in B^{k}(\varepsilon)$ then one gets that $\left(x, d_{x} S(t, u, \eta ; x)\right) \in \operatorname{supp} \chi_{E}^{(u)} \subset p_{0}^{-1}((E-(c+1) \varepsilon, E+(c+1) \varepsilon))$ with $c>0$ as in (13). By (40) and the admissibility assumption (B) it follows that by possibly shrinking $t \neq 0$, we can ensure that there is a constant $C_{0}>0$, such that the non-degeneracy condition

$$
\operatorname{det}\left(d_{u^{\prime}} d_{\eta} \varphi_{t}\left(y, u^{\prime}, u^{\prime \prime}, \eta ; x\right)\right)=|t|^{n}\left(\operatorname{det}\left(d_{u^{\prime}} d_{\eta} p_{u}(x, \eta)\right)+\mathcal{O}\left(t^{2}\right)\right) \geq C_{0}|t|^{n},
$$

holds uniformly for $(u, y, \eta)$ with $(y, \eta) \in \operatorname{supp} \chi_{E}^{(0}$ and $y=d_{\eta} S(t, u, \eta ; x)$. Now, for $u^{\prime \prime}$ fixed and $x \in U$, consider the map

$$
\left(u^{\prime}, y, \eta\right) \longmapsto d_{\eta} \varphi_{t}\left(y, u^{\prime}, u^{\prime \prime}, \eta ; x\right), \quad\left(u^{\prime}, \tau ; y, \eta\right) \in \Gamma_{t, x, u^{\prime \prime}}
$$


We claim that due to the non-degeneracy condition (41), the Lagrangian (40) is a canonical graph. Indeed, equation (41) allows us to apply the Implicit Function Theorem and locally write $u^{\prime}=u^{\prime}(y, \eta)$ satisfying

$$
u^{\prime}=u^{\prime}(y, \eta) \quad \text { when } d_{\eta} \varphi_{t}\left(y, u^{\prime}, u^{\prime \prime}, \eta ; x\right)=0,
$$

for $x \in U$. Then, taking into account that for $x \in U$

$$
d_{\eta} \varphi_{t}\left(y, u^{\prime}, u^{\prime \prime}, \eta ; x\right)=0 \quad \text { when } y=d_{\eta} S\left(t, u^{\prime}, u^{\prime \prime}, \eta ; x\right)
$$

we write $(y, \eta) \in V \times \mathbb{R}^{n}$ as local parametrizing variables for $\Gamma_{t, x, u^{\prime \prime}}$ as in (40) and get

$$
\begin{aligned}
\Gamma_{t, x, u^{\prime \prime}}=\left\{\left(u^{\prime}(y, \eta), d_{u^{\prime}} S\left(t, u^{\prime}(y, \eta), u^{\prime \prime}, \eta ; x\right) ; y, \eta\right):\right. \\
\\
\left.(y, \eta) \in \operatorname{supp} \chi_{E}^{(0)}, y=d_{\eta} S\left(t, u^{\prime}, u^{\prime \prime}, \eta ; x\right)\right\} .
\end{aligned}
$$

For $u^{\prime \prime} \in B^{k-n}(\varepsilon)$ and $x \in U$ define the operators

$$
A_{t, x, u^{\prime \prime}}(h): C^{\infty}(M) \longrightarrow C^{\infty}(M), \quad A_{t, x, u^{\prime \prime}}(h) \stackrel{\text { def }}{=}\left(\widehat{W}_{t, x, u^{\prime \prime}}(h)\right)^{*} \circ\left(\widehat{W}_{t, x, u^{\prime \prime}}(h)\right) .
$$

Since $\widehat{W}_{t, x, u^{\prime \prime}}(h) \in I_{\mathrm{cl}}^{0,-\infty}\left(M \times B^{n}(\varepsilon) ; \Gamma_{t, x, u^{\prime \prime}}\right)$ and the immersed Lagrangian $\Gamma_{t, x, u^{\prime \prime}}$ is a canonical graph, the operator

$$
A_{t, x, u^{\prime \prime}}(h) \in \Psi_{\mathrm{cl}}^{0,-\infty}(M),
$$

for $x \in U$ and $u^{\prime \prime} \in B^{k-n}(\varepsilon)$. From (38) and (44) it follows that

$$
\int_{B^{k}(\varepsilon)}\left|\varphi_{h, t}^{(u)}(x)\right|^{2} d \nu(u)=c_{k}(\varepsilon) \int_{B^{k-n}(\varepsilon)}\left\langle A_{t, x, u^{\prime \prime}}(h) \varphi_{\hbar}, \varphi_{\hbar}\right\rangle_{L^{2}(M)} d u^{\prime \prime}+\mathcal{O}\left(h^{\infty}\right) .
$$

4.1. Proof of Theorem 1. Since $M$ is compact we choose a finite covering

$$
M \subset \bigcup_{j=1}^{N} \mathcal{V}_{x_{j}}
$$

where $x_{j} \in M$ and $\mathcal{V}_{x_{j}}=\mathcal{W}_{x_{j}} \cap U_{x_{j}}$. Here $\mathcal{W}_{x_{j}}$ is given by part (B) of the admissibility condition at $x_{j}$, and $U_{x_{j}}$ is given in Remark 1 .

Fix $j \in\{1, \ldots, N\}$ and let $x \in \mathcal{V}_{x_{j}}$. To prove the first part of Theorem 1 we note that Proposition 5 gives $A_{t, x, u^{\prime \prime}}(h) \in \Psi_{\mathrm{cl}}^{0,-\infty}(M)$. Thus, by $L^{2}$ boundedness there exists a constant $C_{j}=C_{j}\left(\varepsilon, t, E, g_{0}\right)>0$ such that

$$
\left\langle A_{t, x, u^{\prime \prime}}(h) \varphi_{\hbar}, \varphi_{\hbar}\right\rangle_{L^{2}(M)} \leq C_{j}
$$


uniformly in $\left(x, u^{\prime \prime}, h\right) \in \mathcal{V}_{x_{j}} \times B^{k-n}(\varepsilon) \times\left(0, h_{0}\right]$. Therefore, from (8) and (35) one can choose a positive constant $C>0$ so that the first part of the statement of Theorem 1 holds uniformly for $x \in K$, where $K \subset\left(V^{-1}(E)\right)^{c}$ is any compact subset.

To prove the second part of Theorem 1 regarding the odd moments we simply apply Proposition 3 in each neighborhood $\mathcal{V}_{x_{j}}$.

4.2. Proof of Theorem 2. From Proposition 5 and equation (8),

$$
\begin{aligned}
\lim _{h \rightarrow 0^{+}} \operatorname{Var}\left[\operatorname{Re}\left(\varphi_{h, t}^{(\cdot)}(x)\right)\right] & =\lim _{h \rightarrow 0^{+}} \int_{B^{k}(\varepsilon)}\left|\varphi_{h, t}^{(u)}(x)\right|^{2} d \nu(u) \\
& =\lim _{h \rightarrow 0^{+}} c_{k}(\varepsilon) \int_{B^{k-n}(\varepsilon)}\left\langle A_{t, x, u^{\prime \prime}}(h) \varphi_{\hbar}, \varphi_{\hbar}\right\rangle_{L^{2}(M)} d u^{\prime \prime} .
\end{aligned}
$$

Since $\left(\varphi_{h}\right)$ is a quantum ergodic sequence,

$$
\begin{aligned}
& \lim _{h \rightarrow 0^{+}}\left\langle A_{t, x, u^{\prime \prime}}(h) \varphi_{\hbar}, \varphi_{\hbar}\right\rangle_{L^{2}(M)} \\
& \quad=\frac{1}{\left|p_{0}^{-1}(E)\right|} \int_{p_{0}^{-1}(E)} \sigma_{0}\left(A_{t, x, u^{\prime \prime}}(h)\right)(y, \eta) d \omega_{E}(y, \eta) .
\end{aligned}
$$

In addition, following the same argument presented in Corollary 4.2 of [8], the principal symbol can be locally written as

$$
\begin{aligned}
\sigma_{0} & \left(A_{t, x, u^{\prime \prime}}(h)\right)(y, \eta) \\
& =\left|\chi_{E}^{\left(u^{\prime}, u^{\prime \prime}\right)}(x, \eta)\right|^{2} \frac{\left|\operatorname{det}\left(d_{x} \pi G_{\left(u^{\prime}, u^{\prime \prime}\right)}^{-t}(x, \eta)\right)\right|}{\left|\operatorname{det}\left(d_{u^{\prime}} d_{\eta} S\left(t, x, \eta ; u^{\prime}, u^{\prime \prime}\right)\right)\right|} \chi^{2}\left(u^{\prime}, u^{\prime \prime}\right) \\
& =\left|\chi_{E}^{\left(u^{\prime}, u^{\prime \prime}\right)}(x, \eta)\right|^{2} \frac{\left|\operatorname{det}\left(d_{x} \pi G_{\left(u^{\prime}, u^{\prime \prime}\right)}^{-t}(x, \eta)\right)\right|}{|t|^{n}\left|\operatorname{det}\left(d_{u^{\prime}} d_{\eta} p_{\left(u^{\prime}, u^{\prime \prime}\right)}(x, \eta)\right)\right|}(1+\mathcal{O}(t)) \chi^{2}\left(u^{\prime}, u^{\prime \prime}\right)
\end{aligned}
$$

for $u^{\prime}=u^{\prime}(y, \eta)$ parametrizing the Lagrangian $\Gamma_{t, x, u^{\prime \prime}}$ regarded as a canonical graph. The first statement of Theorem 2 then follows by combining (45), (46) and the expression for the principal symbol (47).

The second statement of Theorem 2 about odd moments is a direct application of the second part of Theorem 1 .

\section{Admissible perturbations}

In this section we study the geometry behind the admissibility condition and show that perturbations satisfying such conditions always exist. It is clear that one can always have perturbations satisfying part (B) of the admissibility condition. We therefore 
focus on proving the existence of metric perturbations satisfying condition (A). The symbol $p_{u}: T^{*} M \rightarrow T^{*} M$ defined in (3) has the form

$$
p_{u}(x, \xi)=\sum_{i, j=1}^{n} g_{u}^{i j}(x) \xi_{i} \xi_{j}+V(x)
$$

Write $\mathcal{M}$ for the space of Riemannian metrics on $M$. For each coordinate $u_{s}$ of $u$ define the symmetric tensor $h_{u_{s}} \stackrel{\text { def }}{=} \delta_{u_{s}} g_{u}^{-1}$ and write in local coordinates

$$
h_{u_{s}}=h_{u_{s}}^{i j} d x_{i} \otimes d x_{j}, \quad h_{u_{s}}^{i j} \stackrel{\text { def }}{=} \delta_{u_{s}} g_{u}^{i j}
$$

It is straight forward to check that $\left.\partial_{u_{S}} \partial_{\xi_{i}} p_{u}(x, \xi)\right|_{u=0}=2 \sum_{l=1}^{n} h_{u_{s}}^{l i}(x) \xi_{l}$. Thereby, a metric perturbation satisfies condition (A) provided there exist $c>0$ and an $n$-tuple $u^{\prime}=\left(u_{1}, \ldots, u_{n}\right)$ of coordinates of $u$ so that for all $(x, \xi) \in p_{0}^{-1}(E-c \varepsilon, E+$ $c \varepsilon)$, the matrix $\left(\sum_{l=1}^{n} h_{u_{j}}^{l i}(x) \xi_{l}\right)_{i, j=1, \ldots, n}$ is invertible. By definition, the notion of admissibility depends on the direction, inside the space of symmetric tensors, in which $g_{0}$ is deformed. In what follows we show that the admissibility condition is directly related to performing the deformation $g_{u}$ in sufficiently many volume preserving directions, described below.

Let $\mathcal{P}$ denote the multiplicative group of positive smooth functions on $M$, which we refer to as pointwise conformal deformations. $\mathcal{P}$ acts on $\mathcal{M}$ by multiplication

$$
\mathcal{P} \times \mathcal{M} \longrightarrow \mathcal{M}, \quad(p, g) \longmapsto p g
$$

Given $g_{0} \in \mathcal{M}$, the orbit of $g_{0}$ under $\mathcal{P}$ denoted by $\mathcal{P} \cdot g_{0}$, is a closed submanifold of $\mathcal{M}$ with tangent space at $g_{0}$ given by

$$
T_{g_{0}}\left(\mathcal{P} \cdot g_{0}\right)=\left\{v \in S^{2}(M): v=f g_{0}, f \in C^{\infty}(M, \mathbb{R})\right\} .
$$

Let $\mu$ be a volume form on $M$ and define

$$
\mathcal{N}_{\mu} \stackrel{\text { def }}{=}\left\{g \in \mathcal{M}: \mu=\mu_{g}\right\}
$$

where $\mu_{g}$ denotes the Riemannian volume measure associated to $g$. Pointwise conformal transformations $g_{0} \mapsto f g_{0}$ multiply the volume form $\mu_{g_{0}}(x)$ at a point $x$ by $(f(x))^{n / 2}$. Transverse to the orbit of $g_{0}$ by the action of pointwise conformal transformations is the sub manifold $\mathcal{N}_{\mu_{g_{0}}}$ of all metrics $g$ on $M$ with the fixed volume form; equivalently, the $\operatorname{determinant} \operatorname{det}\left(g_{i j}(x)\right)$ is preserved for all $x$. It is well-known that the tangent space to the space of symmetric matrices with fixed determinant consists of symmetric traceless matrices. Accordingly, it can be shown (cf. [6]) that the tangent space $T_{g_{0}} \mathcal{N}_{\mu_{g_{0}}}$ is given by

$$
T_{g_{0}}\left(\mathcal{N}_{\mu_{g_{0}}}\right)=\left\{v \in S^{2}(M):\left(\operatorname{tr}_{g_{0}} v\right)(x)=0, x \in M\right\}
$$


For every metric $g_{0} \in \mathcal{M}$ the space of symmetric tensors has the pointwise orthogonal splitting

$$
T_{g_{0}} \mathcal{M}=T_{g_{0}}\left(\mathcal{N}_{\mu_{g_{0}}}\right) \oplus T_{g_{0}}\left(\mathcal{P} \cdot g_{0}\right)
$$

where every $v \in S^{2}(M)$ is decomposed as

$$
v=\left(v-\frac{\operatorname{tr}_{g_{0}} v}{n} g_{0}\right)+\frac{1}{n}\left(\operatorname{tr}_{g_{0}} v\right) g_{0}
$$

Let $g_{u}$ be a metric deformation of $g_{0}$; we continue to write $h_{u_{s}}=\delta_{u_{s}} g_{u}^{-1}$. Working in geodesic normal coordinates at $x_{*}$, it is not difficult to show that volumepreserving deformations are characterized by the condition $\operatorname{tr}_{g_{0}^{-1}}\left(h_{u_{s}}(x)\right)=0$ for all $s$. We shall show below that the admissibility condition holds for such deformations.

5.1. Surfaces. On surfaces, we claim that perturbations $g_{u}$ that have two linearly independent $u$-derivatives in the volume preserving directions are admissible.

Proposition 6. Let $\left(M, g_{0}\right)$ be a compact Riemannian surface. Let $E$ be a regular value of $p_{0}$. Suppose $g_{u}$ with $u \in B^{k}(\varepsilon)$ is a perturbation of $g_{0}$ such that there exist two coordinates $u^{\prime}=\left(u_{1}, u_{2}\right)$ of $u$ for which $h_{u_{1}}(x)$ and $h_{u_{2}}(x)$, as defined in (48), are linearly independent tensors with $\operatorname{tr}_{g_{0}^{-1}}\left(h_{u_{1}}\right)(x)=\operatorname{tr}_{g_{0}^{-1}}\left(h_{u_{2}}\right)(x)=0$ at some $x \notin V^{-1}(E)$. Then, for $\varepsilon$ small enough, the perturbation $g_{u}$ satisfies part (A) of the admissibility condition at $x$.

Proof. Let $x_{*} \in M$ be such that $x$ belongs to a geodesic ball cantered at $x_{*}$, and consider normal coordinates at $x_{*}$. In these coordinates, $g_{0_{i j}}(x)=\delta_{i j}+\mathcal{O}\left(|x|^{2}\right)$ for $x$ being at a small distance $|x|$ from $x_{*}$. Therefore, since $\operatorname{tr}_{g_{0}^{-1}}\left(h_{u_{s}}\right)(x)=0$ for $s=1,2$, we have $h_{u_{s}}^{11}(x)=-h_{u_{s}}^{22}(x)+\mathcal{O}\left(|x|^{2}\right)$ for $s=1,2$. It is straight forward to check

$$
\operatorname{det}\left(\sum_{j=1}^{2} h_{u_{s}}^{i j}(x) \xi_{j}\right)_{s, i=1,2}=|\xi|_{g_{0}(x)}^{2}\left(\operatorname{det}\left(\begin{array}{ll}
h_{u_{1}}^{11}(x) & h_{u_{2}}^{11}(x) \\
h_{u_{1}}^{12}(x) & h_{u_{2}}^{12}(x)
\end{array}\right)+\mathcal{O}\left(|x|^{2}\right)\right)
$$

Since we are only interested in what happens when $|\xi|_{g_{0}(x)}^{2}+V(x)=E+\mathcal{O}(\varepsilon)$, the result follows from the assumption $V(x) \neq E$ and the fact that $h_{u_{1}}(x)$ and $h_{u_{2}}(x)$ are linearly independent tensors.

5.2. Manifolds. In what follows we show that on an $n$-dimensional manifold we can always have admissible perturbations. 
Let $M$ be an $n$-dimensional compact manifold and fix $x_{*} \in M$. Consider a geodesic normal coordinate system at $x_{*}$. We shall consider deformations of the reference metric $g_{0}$ that, as in the surface case, preserve the volume form. Infinitesimally, as explained in (50), the corresponding quadratic form is given by a traceless symmetric matrix. The space of traceless symmetric tensors at $x \in M$ ) has dimension

$$
\kappa_{n} \stackrel{\text { def }}{=} \frac{n^{2}+n-2}{2},
$$

and the basis of the space of such forms is given by

$$
\xi_{1}^{2}-\xi_{i}^{2}, 2 \leq i \leq n ; \quad \text { and } \quad \xi_{j} \xi_{k}, 1 \leq j<k \leq n
$$

for $\xi=\left(\xi_{1}, \ldots, \xi_{n}\right) \in T_{x}^{*} M$; we denote these polynomials evaluated at $x=x_{*}$ by $q_{j}(\xi)$ for $j=1, \ldots, \kappa_{n}$.

Note that since we are using normal coordinates centered at $x_{*}$ then $|\xi|_{g_{0}\left(x_{*}\right)}^{2}=$ $\sum_{i} \xi_{i}^{2}$, and remark that the polynomials $q_{j}(\xi)$ form a basis in the space of spherical harmonics of degree two on the sphere $S_{x_{*}}^{n-1}=\left\{\xi \in T_{x_{*}}^{*} M:|\xi|_{g_{0}\left(x_{*}\right)}=1\right\}$ with the round metric $g_{S_{x *}^{n-1}}$. In the proof we shall use a computation showing that the round metric on $S_{x_{*}}^{n-1}$ is an extremal for the second eigenvalue of the Laplacian in the space of nearby Riemannian metrics. We refer to [7], [12], and [14] and references therein for a description of general theory of such metrics.

Below we summarize several well-known facts about extremal metrics. Let $g_{0}$ be an extremal metric on a compact $d$-dimensional manifold $N$. Then

a) if $\left(N, g_{0}\right)$ is a homogeneous space (e.g. a round $\left.S^{n-1}\right)$, then the metric $g_{0}$ is extremal for all eigenvalues of the Laplacian $\Delta_{g_{0}}$;

b) if $g_{0}$ is extremal for an eigenvalue $\lambda$ (of multiplicity $m$ ), and $\varphi_{1}, \ldots, \varphi_{m}$ form an orthonormal basis of the corresponding eigenspace $E_{\lambda}$, then

$$
\sum_{j=1}^{m} d \varphi_{j} \otimes d \varphi_{j}=(\lambda / 2) g_{0} .
$$

We remark that one can show that $m>d$ in (52).

Claim 1. Assume that $\left\{\varphi_{1}, \ldots, \varphi_{m}\right\}$ satisfy (52). Then the (Jacobian) matrix

$$
\frac{\partial\left(\varphi_{1}, \ldots, \varphi_{m}\right)}{\partial\left(x_{1}, \ldots, x_{d}\right)}
$$

has the maximal possible rank $d$.

Proof. Assume that the rank is less than $d$. Than the quadratic form $\sum_{j=1}^{m} d \varphi_{j} \otimes d \varphi_{j}$ cannot be positive-definite; however by assumption it is proportional to the positivedefinite Riemannian metric $g_{0}$ on $N$. Contradiction finishes the proof. 
The existence of admissible perturbations will follow from Claim 1.

We shall show that a metric perturbation is admissible if it is of the form

$$
g_{u}^{-1}(x)=g_{0}^{-1}(x)+\sum_{j=1}^{\kappa_{n}} u_{j} h_{j}(x),
$$

where $h_{j}(x)(\xi, \xi)$ are homogeneous of degree 2 in the $\xi$ variables and are required to satisfy the following conditions:

(a) $h_{j}\left(x_{*}\right)(\xi, \xi)=q_{j}(\xi), 1 \leq j \leq \kappa_{n}$;

(b) $h_{j}$ are $C^{2}$ tensors.

Proposition 7. Let a perturbation of $g_{u}$ of $g_{0}$ have the form (53) with $h_{j}$ satisfying conditions (a) and (b). Then there exist $\varepsilon>0$ and $\delta>0$ such that for all $u \in B^{n}(\varepsilon)$ the perturbation $g_{u}$ satisfies part (A) of the admissibility condition at $x$ for $x \in B\left(x_{*}, \delta\right)$.

Proof. Clearly, $\partial_{u_{j}}\left(|\xi|_{g_{u}\left(x_{*}\right)}\right)=q_{j}(\xi)$, and hence the $j$-th column (say) of the mixed hessian matrix $d_{\xi} d_{u} p_{u}\left(x_{*}, \xi\right)$ corresponds to the gradient $d_{\xi} q_{j}(\xi)$.

Now, since the sphere $S_{x_{*}}^{n-1}$ is a homogeneous space, the round metric $g_{S_{x_{*}}^{n-1}}$ is a critical metric for the corresponding eigenvalue functional $g \mapsto \lambda(g) \cdot \operatorname{Vol}(g)^{2 / n}$, where $\lambda$ denotes the second positive eigenvalue (without multiplicity) of the Laplacian.

By (52), see [7], [12], and [14], the $L^{2}$-normalized basis of the eigenspace $E(\lambda)$ (which can be chosen as $\left\{q_{1}(\xi), \ldots, q_{\kappa_{n}}(\xi)\right\}$ in our case) satisfies

$$
\sum_{j=1}^{\kappa_{n}} d_{\xi} q_{j} \otimes d_{\xi} q_{j}=c \lambda g_{S_{x *}^{n-1}}, \quad c \neq 0 .
$$

By Claim 1, the subspace spanned by $d_{\xi} q_{1}(\xi), \ldots, d_{\xi} q_{\kappa_{n}}(\xi)$ has the full dimension $n-1$ in $T_{\xi}^{*}\left(S_{x_{*}}^{n-1}\right)$ at any point $\xi \in S_{x_{*}}^{n-1}$. This shows that $\left\{d_{\xi} q_{j}(\xi): 1 \leq j \leq \kappa_{n}\right\}$ span the full $T_{\xi}^{*}\left(S_{x_{*}}^{n-1}\right)$, which proves the required non-degeneracy condition.

Next, since $h_{j}$ are $C^{2}$ in $(x, \xi)$ by condition (b), the rank of $d_{\xi} d_{u} p_{u}(x, \xi)$ changes continuously in $x$ and so is equal to $n-1$ for $x \in B\left(x_{*}, \delta\right)$ on $M$ for some $\delta>0$.

\section{References}

[1] M. Berry, Regular and irregular semiclassical wavefunctions. J. Phys. A 10 (1977), 2083-2091. MR 0489542 Zbl 0377.70014

[2] N. Burq, P. Gérard, and N. Tzvetkov, Restrictions of the Laplace-Beltrami eigenfunctions to submanifolds. Duke Math. J. 138 (2007), 445-486. MR 2322684 Zbl 1131.35053

[3] F. Cucchietti, H. Pastawski, and D. Wisniacki, Decoherence as decay of the Loschmidt echo in a Lorentz gas. Phys. Rev. E 65 (2002). Article Id. 045206. 
[4] J. Duistermaat, Fourier integral operators. Progress in Mathematics 130. Birkhäuser Boston, Boston, MA, 1996. MR 1362544 Zbl 0841.35137

[5] S. Dyatlov and M. Zworski, Quantum ergodicity for restrictions to hypersurfaces. Nonlinearity 26 (2013), 35-52. MR 3001760 Zbl 06163460

[6] D. Ebin, The manifold of Riemannian metrics. In Global Analysis (Proc. Sympos. Pure Math., Vol. XV, Berkeley, Calif., 1968). American Mathematical Society, Providence, R.I., 1968, 11-40. MR 0267604 Zbl 0205.53702

[7] A. El Soufi and S. Ilias. Laplacian eigenvalue functionals and metric deformations on compact manifolds. J. Geom. Phys. 58 (2008), 89-104. MR 2378458 Zbl 1137.49040

[8] S. Eswarathasan and J. Toth. Averaged pointwise bounds for deformations of Schrödinger eigenfunctions. Annales Henri Poincaré 14 (2013), 611-637. MR 3035641

Zbl 1263.81186

[9] D. Hejhal and B. Rackner. On the topography of Maass waveforms for PSL(2, Z). Experiment. Math. 1 (1992), 275-305. MR 1257286 Zbl 0813.11035

[10] P. Jacquod, P. Silvestrov, and C. Beenakker, Golden rule decay versus Lyapunov decay of the quantum Loschmidt echo. Phys. Rev. E 64 (2001). Article Id. 055203.

[11] R. Jalabert and H. Pastawski, Environment-independent decoherence rate in classically chaotic systems. Physical review letters 86 (2001), 2490-2493.

[12] N. Nadirashvili, Berger's isoperimetric problem and minimal immersions of surfaces. Geom. Funct. Anal. 6 (1996), 877-897. MR 1415764 Zbl 0868.58079

[13] A. Peres, Stability of quantum motion in chaotic and regular systems. Phys. Rev. A (3) 30 (1984), 1610-1615. MR 0762403

[14] T. Takahashi, Minimal immersions of riemannian manifolds. J. Math. Soc. Japan 18 (1966), 380-385. MR 0198393 Zbl 0145.18601

[15] J. Toth and S. Zelditch, Quantum ergodic restriction theorems: manifolds without boundary. Geom. Funct. Anal. 23 (2013), 715-775. MR 3053760 Zbl 06180408

[16] D. Wisniacki and D. Cohen, Quantum irreversibility, perturbation independent decay, and the parametric theory of the local density of states. Phys. Rev. E 66 (2002) 046-209.

[17] M. Zworski, Semiclassical analysis. Graduate Studies in Mathematics 138. American Mathematical Society, Providence, RI, 2012. MR 2952218 Zbl 1252.58001

Received March 20, 2013

Yaiza Canzani, Department of Mathematics and Statistics, McGill University, 805 Sherbrooke Str. West, Montréal QC H3A 2K6, Canada.

E-mail: canzani@math.mcgill.ca

Dmitry Jakobson, Department of Mathematics and Statistics, McGill University, 805 Sherbrooke Str. West, Montréal QC H3A 2K6, Canada.

E-mail: jakobson@math.mcgill.ca

John Toth, Department of Mathematics and Statistics, McGill University, 805 Sherbrooke Str. West, Montréal QC H3A 2K6, Canada.

E-mail: jtoth@math.mcgill.ca 